\title{
Sequential Observation of 2,6-Dimethylaniline-Induced Nasal Lesions in a Rat Two- stage Nasal Carcinogenesis Model after Initiation with N-bis(2-Hydroxypropyl) Nitrosamine
}

\author{
Takatoshi KOUJITANI', Kazuo YASUHARA ${ }^{1)}$, Takako IKEDA',2), Takayoshi IMAZAWA ${ }^{1)}$, Toru TAMURA ${ }^{11}$, \\ Kaoru TOYOSAWA ${ }^{3)}$, Akinori SHIMADA ${ }^{4)}$, Masao HIROSE ${ }^{1)}$ and Kunitoshi MITSUMORI ${ }^{1) *}$ \\ ${ }^{1)}$ Division of Pathology, National Institute of Health Sciences, 1-18-1 Kamiyoga, Setagaya-ku, Tokyo 158-8501, ${ }^{2)}$ Showa Women's \\ University, 1-7 Taishido, Setagaya-ku, Tokyo 154-8533, ${ }^{3)}$ Department of Developmental Research Laboratories, Dainippon \\ Pharmaceutical Co., Ltd., 33-94 Enoki-cho, Suita-shi, Osaka 564-0053, and ${ }^{4}$ Department of Veterinary Pathology, Tottori University, \\ 4-101 Koyama-cho Minami, Tottori 680-0945, Japan
}

(Received 21 January 2000/Accepted 25 March 2000)

ABSTRACT. Male F344 rats received diet containing 3,000 ppm 2,6-dimethylaniline (DMA) after initiation with a single subcutaneous injection of 2,400 mg/kg of N-bis(2-hydroxypropyl)nitrosamine (DHPN), and histological and electron microscopic examinations of the nasal cavity were performed at 4, 13, 26 and 52 weeks to examine sequential changes induced by DMA. Severe atrophy of Bowman's glands and epithelial disarrangement were apparent from week 4, followed by dilatation and/or proliferation of Bowman's glands, degeneration of epithelial cells, and proliferation of undifferentiated epithelial cells from week 13. Focal glandular hyperplasias, dysplastic foci, and adenomas were observed from week 26, and carcinomas at 52 week. These nasal lesions were mostly evident in the olfactory mucosa in the nasal cavity, and their severity and/or incidences, other than atrophy of Bowman's glands, increased with the treatment period. Electron microscopically, carcinoma cells demonstrated desmosomes, dense secretory granules identical to those in normal Bowman's glands, a basement membrane, and microvilli. These results suggest that Bowman's glands are the target of DMA, giving rise to nasal carcinomas after DHPN-initiation.

KEY WORDS: carcinogenicity, 2,6-dimethylaniline, nasal cavity, N-bis(2-hydroxypropyl)nitrosamine, rat.

J. Vet. Med. Sci. 62(7): 751-756, 2000

2,6-Dimethylaniline (DMA) has been shown to cause a significant increase in the incidence of nasal adenomas and carcinomas in both male and female rats when administered in the diet at 3,000 ppm for 2 years [10]. In mutagenicity tests, DMA proved positive in mouse lymphoma cells, inducing sister chromatid exchange and chromosomal aberrations, but negative in Ames tests $[3,4,12,17]$. DMA is one of the major metabolites of xylazine, an $\alpha_{2}$-adrenergic agonist with sedative, muscle relaxant and analgesic properties that is commonly used in veterinary medicine $[15,16]$.

In our previous two-stage nasal carcinogenesis study of DMA in rats using N-bis(2-hydroxypropyl)nitrosamine (DHPN) as an initiator, the development of glandular hyperplasias, dysplastic foci, and epithelial tumors in the nasal cavity was clearly enhanced by dietary administration at the dose of 3,000 ppm for 52 weeks [6]. On the other hand, no tumor promoting effects of xylazine in the nasal cavity were demonstrated in animals fed 1,000 ppm (maximum tolerated dose) for 52 weeks after DHPN initiation [7].

Since the relationship of non-neoplastic changes such as glandular hyperplasias and dysplastic foci as well as the origin of epithelial tumors in the nasal cavity remains undetermined, the current study was performed to investigate sequential morphological changes induced by DMA in the nasal cavity in the DHPN-initiated two-stage carcinogenesis model in rats.

* Correspondence to: Mitsumori, K., Division of Pathology, National Institute of Health Sciences, 1-18-1 Kamiyoga, Setagaya-ku, Tokyo 158-8501, Japan.

\section{MATERIALS AND METHODS}

Animals and Chemicals: A total of 76 male F344 rats were obtained from Charles River Japan Inc., Kanagawa, Japan, at 4 weeks of age. They were housed 3 or 4 to a polycarbonate cage with wood chips for bedding under barrier system conditions (room temperature, $23 \pm 2{ }^{\circ} \mathrm{C}$; relative humidity, $55 \pm$ 5\%; air change, 18 times/hr; 12 hr-light/12 hr-dark cycle). They were quarantined for 1 week in the animal room, and given pulverized basal diet (Oriental CRF-1, Oriental Yeast Co., Tokyo, Japan) and tap water ad libitum.

DMA (purity, 99\%) and DHPN were obtained from Wako Pure Chemical Industries, Ltd., (Osaka, Japan) and Nacalai Tesque, Inc., (Kyoto, Japan), respectively.

Experimental design: The male F344 rats were randomly subdivided into a DHPN/DMA-treated group $(\mathrm{N}=50)$ and an untreated group $(\mathrm{N}=26)$, respectively given a single subcutaneous injection of $2,400 \mathrm{mg} / \mathrm{kg}$ of DHPN in isotonic sodium chloride solution and the vehicle. Starting one week after this initiation treatment, diet containing 3,000 ppm DMA was given to the DHPN/DMA-treated group. The chemical was first dissolved in corn oil (Sigma Aldrich Japan, Tokyo, Japan) and then admixed with the basal diet at $10 \mathrm{ml}$ corn oil per $\mathrm{kg}$. Test diet was prepared every week and stored in a refrigerator (temperature, $4^{\circ} \mathrm{C}$ ) before use. Stability data showed that DMA level in the diet stored at room temperature for 1 week was $87.4 \%$ compared with that sampled at the diet preparation. So diet was changed twice a week during the experimental period. Controls received the basal diet throughout the 
study.

Necropsy and light microscopic examination: Dead or moribund animals during the experimental period were subjected to a complete necropsy as soon as they were found. Surviving animals were killed under ether anesthesia by exsanguination from the abdominal aorta at weeks 4, 13, 26 and $52(3,7,10$ and 30 rats respectively, for the DHPN/DMA and $4,4,8$ and 10 for the untreated group), and similarly necropsied. Those in the untreated group were 4, 4, 8 and 10, respectively. Nasal cavities were fixed with $10 \%$ neutral buffered formalin after flushing the fixative into the posterior opening of the nasopharynx with a syringe. Following fixation, the heads were decalcified in a 5\% formic acid solution. Using the upper incisor teeth, incisive papilla, and first upper molar tooth as guides for trimming, six transverse sections including levels I, II, and III through the nasal cavity were prepared based on the method of Nagano et al. [9]. After postfixation, the nasal sections were embedded in paraffin, sectioned at 4-5 $\mu \mathrm{m}$, and stained with hematoxylin and eosin (HE) for light microscopic examination.

Electron microscopic examination: At necropsy, some carcinomas from moribund or sacrificed animals were used for ultrastructural studies. The selected tissues were fixed in fixative $(2.5 \%$ gultaraldehyde and $4.0 \%$ paraformaldehyde in $0.1 \mathrm{M}$ phosphate buffer) for $2 \mathrm{hr}$ at $4^{\circ} \mathrm{C}$, post-fixed in phos- phate-buffered $1.0 \%$ osmium tetroxide for $1 \mathrm{hr}$, dehydrated through a series of alcohols and embedded in Epon 812 . Ultrathin sections were contrasted with uranyl acetate and lead citrate, and examined under an electron microscope (JEM 1200EX, JEOL or H-7000, Hitachi Ltd., Tokyo, Japan).

\section{RESULTS}

Three DHPN/DMA-treated animals showed a decrease in locomotor activity and swelling of the dorsal area of the nose from week 40 and four DHPN/DMA-treated rats died or were killed in extremis because of the development of nasal cavity tumors.

Histopathologically, various changes consisting of atrophy, dilatation and/or proliferation of Bowman's glands, epithelial disarrangement, degeneration of epithelial cells, proliferation of undifferentiated epithelial cells, focal glandular hyperplasias, dysplastic foci, adenomas, and carcinomas were observed in DHPN/DMA-treated (Tables 1 and 2), but not in untreated rats. These nasal lesions were mostly observed in the olfactory mucosa in the nasal cavity. At 4 weeks, severe atrophy of Bowman's glands and slight epithelial disarrangement were observed in all 3 rats sacrificed, and such changes were consistently present thereafter. Severe atrophy of Bowman's glands was characterized by disappearance of Bow-

Table 1. Histopathological findings for the olfactory mucosa of rats treated with DMA for $4,13,26$ or 52 weeks after DHPN initiation

\begin{tabular}{lcccc}
\hline \multirow{2}{*}{ Histopathological findings } & \multicolumn{4}{c}{ DHPN 2,400 mg/kg (i.p.) + DMA 3,000 ppm } \\
\cline { 2 - 5 } & week 4 & week 13 & week 26 & week 52 \\
\hline Atrophy of Bowman's glands & $3 / 3^{\mathrm{a}}$ & $7 / 7$ & $10 / 10$ & $30 / 30$ \\
Dilatation of Bowman's glands & $0 / 3$ & $7 / 7$ & $10 / 10$ & $30 / 30$ \\
Proliferation of Bowman's glands & $0 / 3$ & $7 / 7$ & $10 / 10$ & $30 / 30$ \\
Epithelial disarrangement & $3 / 3$ & $7 / 7$ & $10 / 10$ & $30 / 30$ \\
Degeneration of epithelial cells & $0 / 3$ & $7 / 7$ & $10 / 10$ & $30 / 30$ \\
Proliferation of undifferentiated & & & & \\
$\quad$ epithelial cells & $0 / 3$ & $7 / 7$ & $10 / 10$ & $30 / 30$ \\
Focal glandular hyperplasias & $0 / 3$ & $0 / 7$ & $7 / 10$ & $30 / 30$ \\
Dysplastic foci & $0 / 3$ & $0 / 7$ & $1 / 10$ & $10 / 30$ \\
Adenomas & $0 / 3$ & $0 / 7$ & $1 / 10$ & $8 / 30$ \\
Carcinomas & $0 / 3$ & $0 / 7$ & $0 / 10$ & $10 / 30$ \\
\hline
\end{tabular}

a) Number of animals with lesions/No. of animals examined.

Table 2. Severity of non-neoplastic lesions of the olfactory mucosa in rats treated with DMA for $4,13,26$ or 52 weeks after DHPN initiation

\begin{tabular}{|c|c|c|c|c|}
\hline \multirow{2}{*}{ Histopathological findings } & \multicolumn{4}{|c|}{ DHPN 2,400 mg/kg (i.p.) + DMA 3,000 ppm } \\
\hline & week 4 & week 13 & week 26 & week 52 \\
\hline Atrophy of Bowman's glands & +++ & +++ & +++ & +++ \\
\hline Dilatation of Bowman's glands & - & + & + & + \\
\hline Proliferation of Bowman's glands & - & + & ++ & ++ \\
\hline Epithelial disarrangement & + & + & ++ & +++ \\
\hline Degeneration of epithelial cells & - & + & + & + \\
\hline $\begin{array}{l}\text { Proliferation of undifferentiated } \\
\text { epithelial cells }\end{array}$ & - & + & + & ++ \\
\hline Focal glandular hyperplasias & - & - & + & +++ \\
\hline
\end{tabular}

-, Negative; +, Slight change; ++, Moderate change; +++, Severe change. 

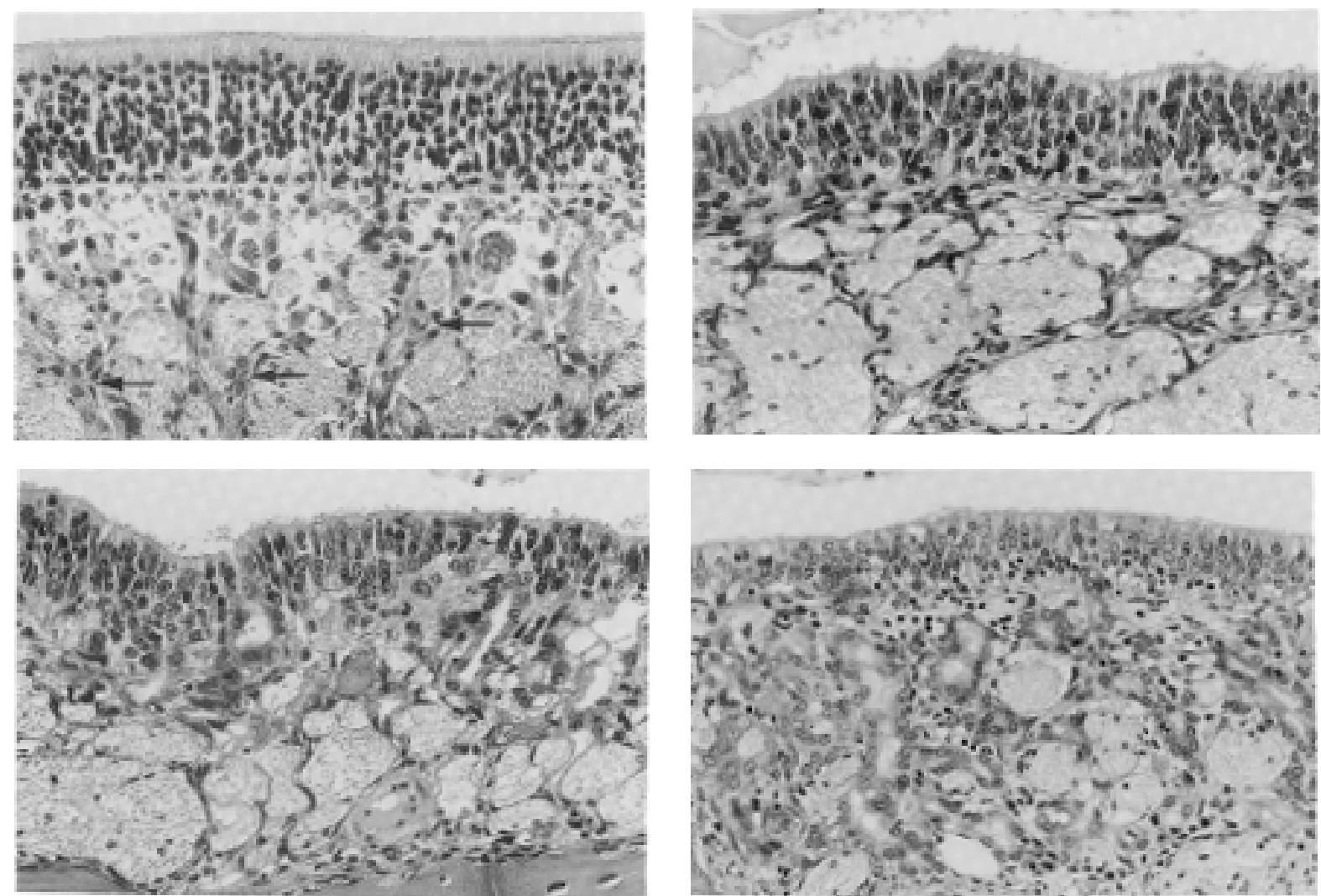

Fig. 1. Normal structure of the olfactory mucosa of an untreated rat killed at week 4. Note many Bowman's glands (arrows) in the lamina propria. HE stain. $\times 300$.

Fig. 2. Atrophy of Bowman's glands in the lamina propria of a DHPN/DMA-treated rat killed at week 4. HE stain. $\times 300$.

Fig. 3. Slight dilatation and proliferation of Bowman's glands, and epithelial disarrangement in a DHPN/DMA-

Fig. 1 Fig. 2

Fig. 3 Fig. 4 treated rat killed at week 13 . HE stain. $\times 300$.

Fig. 4. Moderate proliferation of Bowman's glands consisting of cuboidal cells in a DHPN/DMA-treated rat killed at week 52. HE stain. $\times 240$.

man's glands in the lamina propria (Figs. 1 and 2). In addition, dilatation and/or proliferation of Bowman's glands, degeneration of epithelial cells, and proliferation of undifferentiated epithelial cells were observed in all rats at week 13, and thereafter (Figs. 3 and 4). Proliferation of Bowman's glands was characterized by increased numbers of Bowman's gland consisting of cuboidal cells in the lamina propria of olfactory mucosa (Fig. 4). Proliferation of undifferentiated epithelial cells was characterized by small nests of cuboidal cells with ovoid nuclei at the bottom of the olfactory epithelia. Focal glandular hyperplasias, dysplastic foci, and adenomas were observed from week 26, and carcinomas at week 52 (Figs. 4 7 ). The focal glandular hyperplasias were composed of small nests of glandular structures consisting of cuboidal cells with uniform ovoid nuclei, frequently accompanied by inflammatory cell infiltration (Fig. 5). Dysplastic foci were small solid nests of cuboidal to pleomorphic cells with hyperchromatic nuclei and basophilic cytoplasm in the lamina propria (Fig. 6). Adenomas showed glandular and/or papillary growth pattern of well-differentiated cuboidal tumor cells with glandular structures, projected into the nasal lumen (Fig. 7). Carcinomas were characterized by expansive solid and/or glandular growth of pleomorphic tumor cells with basophilic cytoplasm and bizarre and/or hyperchromatic nuclei, with frequent mitotic figures (Fig. 8). The severity and/or incidences of these nasal changes, except for atrophy of Bowman's glands, increased with the treatment period (Tables 1 and 2).

Electron microscopically, desmosomes were often observed between the tumor cells of carcinomas, along with dense secretory granules (200-800 $\mathrm{nm}$ in diameter) typical of normal Bowman's glands (Figs. 9a, 9b), and a basement membrane around the glandular structures (Fig. 9a). The tumor cells forming lumina possessed microvilli projecting into the lumen and numerous dense secretory granules in their cytoplasm (Fig. 9b).

\section{DISCUSSION}

The olfactory epithelium of the nasal cavity is of pseudostratified columnar type, consisting of sustentacular, sensory, 

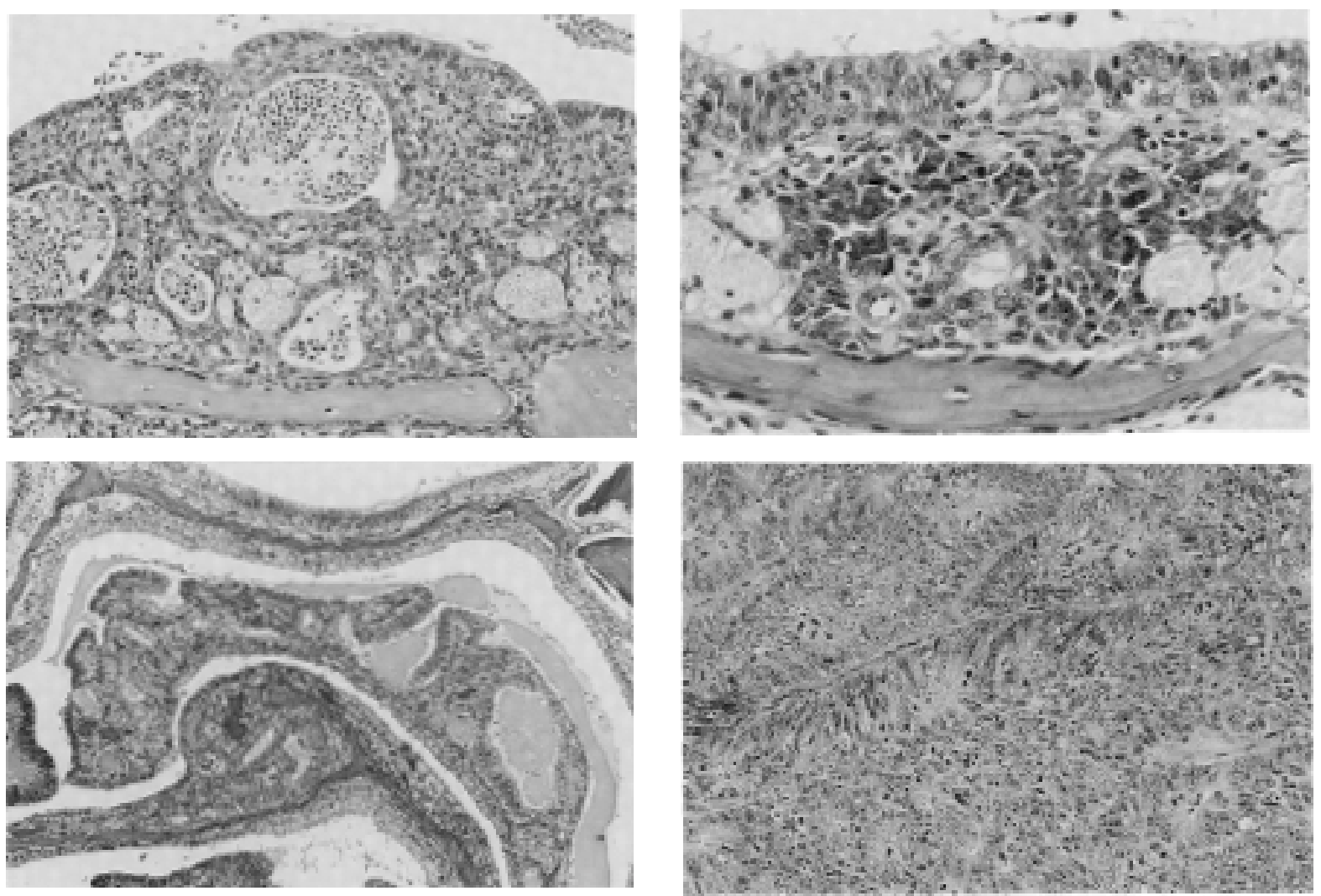

Fig. 5. Focal glandular hyperplasia consisting of cuboidal cells with uniform ovoid nuclei in a DHPN/DMAtreated rat killed at week 52 . HE stain. $\times 150$.

Fig. 6. A dysplastic focus presenting as a small solid nest of cuboidal to pleomorphic cells with hyperchromatic nuclei and basophilic cytoplasm in the lamina propria of a DHPN/DMA-treated rat killed at week 52. HE stain. $\times 300$.

Fig. 7. Adenoma showing a papillary and glandular growth pattern of cuboidal tumor cells in a DHPN/DMAtreated rat killed at week 52. HE stain. $\times 60$.

Fig. 8. Carcinoma showing a glandular growth pattern of pleomorphic tumor cells with bizarre and hyperchromatic nuclei in a DHPN/DMA-treated rat killed at week 52. Mitotic figures are frequently observed. HE stain. $\times 150$.

and basal cells $[2,5]$. The sustentacular cells are considered as supporting cells for the sensory bipolar neurons, while the basal cells are generally considered as stem cells for the regenerating epithelium $[2,5]$. The lamina propria of the olfactory region contains prominent Bowman's glands, and ducts of Bowman's glands traverse the basal lamina, extend through the olfactory epithelium, and open on the luminal surface $[2$, 5].

In the present study, the nasal lesions related to the DHPN/ DMA treatment were mainly observed in the olfactory region in the nasal cavity. Severe atrophy of Bowman's glands and epithelial disarrangement were observed from week 4 . In addition, dilatation and/or proliferation of Bowman's glands, degeneration of epithelial cells, and proliferation of undifferentiated epithelial cells were observed from week 13. Focal glandular hyperplasias, dysplastic foci, and adenomas were observed from week 26, and carcinomas at 52 week. The severity and/or incidences of these nasal lesions, except atrophy of Bowman's glands, increased with the treatment period.
Severe atrophy of Bowman's glands was observed from the early stage in this study. In addition, our preliminary study revealed that atrophy of Bowman's glands in the rat nasal cavity had been induced by the administration of DMA in the diet at 3,000 ppm for 4 weeks (unpublished data). Bowman's glands are clearly targets of DMA. In addition, since proliferation of Bowman's glands morphologically resembled that of focal glandular hyperplasia, the two are likely to be histogenetically related. Carcinomas are considered to arise from dysplastic foci and adenomas through focal glandular hyperplasia, considering their morphological similarities. Atrophy of Bowman's glands resulting in epithelial disarrangement and degeneration of epithelial cells appeared to be followed by proliferation of undifferentiated epithelial cells at the bottom of the olfactory epithelia, where the basal stem cells are located $[2,5]$. In recent experimental studies in which mice were given a single intravenous injection of 2,6-(diCl- $\mathrm{MeSO}_{2}-$ $\mathrm{B})$ and rats were subjected to a single oral administration or 5day inhalation exposure of type IV phosphodiesterase inhibi- 
$\mathbf{a}$

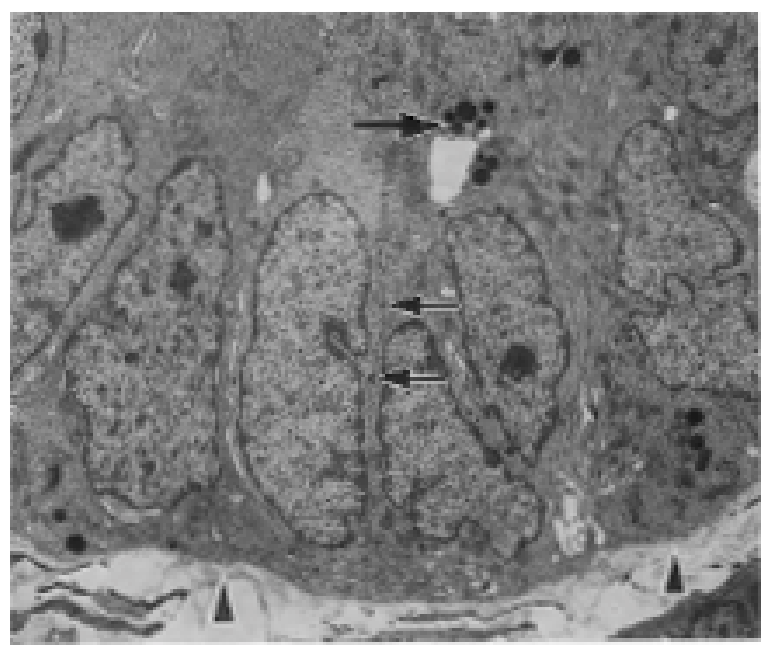

b

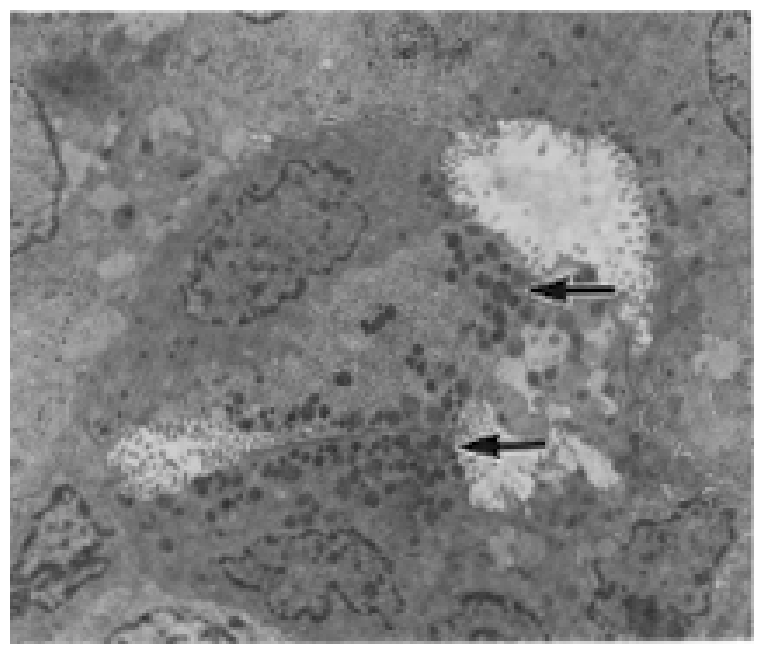

Fig. 9. Electron micrographs of tumor cells of a carcinoma showing glandular structures in a DHPN/DMA-treated rat killed at week 52. (a) Tumor cells have a basement membrane (arrowheads) and desmosomes (small arrows). Note small numbers of dense secretory granules (large arrow) within the cytoplasm that are quite identical to those in normal Bowman's glands. $\times 3,000$.

(b) Tumor cells have numerous dense secretory granules (large arrows). Note microvilli projecting into the lumen. $\times 3,600$.

tor, it has been found that degeneration, necrosis and/or loss of Bowman's glands are followed by disorganization of olfactory epithelium $[1,11]$. Therefore, the epithelial changes of olfactory mucosa observed in our study might be considered secondary to the atrophy of Bowman's glands.

Olfactory mucosal neoplasms in rodents are generally thought to arise from olfactory epithelial elements, with Bowman's glands as the most frequent site of origin [8]. The carcinomas in our study histopathologically resembled those found in the 2-year carcinogenicity study of DMA in rats [10]. The desmosomes apparent between the carcinoma cells, and the presence of dense secretory granules, identical to those in normal Bowman's glands along with the basement membrane and microvilli, indicate an epithelial origin. Sensory cells have olfactory vesicles with cilia that rise above the apical surface, and basal cells have prominent intermediate filaments. In addition, sustentacular cells have microvilli on the apical border and abundant smooth endoplasmic reticulum [5, 13]. In addition to large secretory granules and laminated smooth endoplasmic reticulum [13], the ductal and acinar cells of Bowman's glands have microvilli [14]. Therefore, the carcinomas observed in the present study could very likely have arisen from the Bowman's glands. More detailed immunohistochemical and ultrastructural investigations on the carcinomas, dysplastic foci, and glandular hyperplasia of rats induced by DHPN/DMA treatment are now in progress to clarify the cell of origin and the relationship between these hyperplastic and neoplastic lesions.

ACKNOWLEDGMENTS. This study was supported in part by a Grant-in-Aid for Food Hygiene from the Ministry of Health and Welfare of Japan.

\section{REFERENCES}

1. Bahrami, F., Brittebo, E. B., Bergman, Å., Larsson, C. and Brandt, I. 1999. Localization and comparative toxicity of methylsulfonyl-2,5- and 2,6-dichlorobenzene in the olfactory mucosa of mice. Toxicol. Sci. 49: 116-123.

2. Boorman, G. A., Morgan, K. T. and Uriah, L. C. 1990. Nose, larynx, and trachea. pp. 315-337. In: Pathology of the Fischer Rat (Boorman, G. A., Eustis, S. L., Elwell, M. R., Montgomery Jr., C. A. and MacKenzie, W. F. eds.), Academic Press Inc., San Diego.

3. Florin, I., Ritberg, L., Curvall, M. and Enzell, C. 1980. Screening of tobacco smoke constituents for mutagenicity using the Ames' test. Toxicology 18: 219-232.

4. Galloway, S. M., Armstrong, M. J., Reuben, C., Colman, S., Brown, B., Cannon, C., Bloom, A. D., Nakamura, F., Ahmed, N., Duk, S., Rimpo, J., Margolin, B. H., Resnick, M. A., Anderson, B. and Zeiger, E. 1987. Chromosome aberrations and sister chromatid exchanges in Chinese hamster ovary cells: Evaluations of 108 chemicals. Environ. Mol. Mutagen. 10: 1175.

5. Harkema, J. R. and Morgan, K. T. 1996. Normal morphology of the nasal passages in laboratory rodents. pp. 3-17. In: Monographs on Pathology of Laboratory Animals, Respiratory System, 2nd ed. (Jones, T. C., Dungworth, D. L. and Mohr, U. eds.), Springer-Verlag, Berlin.

6. Koujitani, T., Yasuhara, K., Kobayashi, H., Shimada, A., Onodera, H., Takagi, H., Hirose, M. and Mitsumori, K. 1999. Tumor-promoting activity of 2,6-dimethylaniline in a twostage nasal carcinogenesis model in N-bis(2-hydroxypropyl)nitrosamine-treated rats. Cancer Lett. 142: 161-171.

7. Koujitani, T., Yasuhara, K., Kobayashi, H., Shimada, A., Onodera, H., Takagi, H., Tamura, T., Hirose, M. and Mitsumori, K. 1999. Absence of tumor-promoting activity of xylazine in a two-stage nasal carcinogenesis model in N-bis(2hydroxypropyl)nitrosamine-treated rats. J. Toxicol. Pathol. 12: 203-208. 
8. Morgan, K. T. and Harkema, J. R 1996. Nasal neoplasia. pp. 87-104. In: Monographs on Pathology of Laboratory Animals, Respiratory System, 2nd ed. (Jones, T. C., Dungworth, D. L. and Mohr, U. eds.), Springer-Verlag, Berlin.

9. Nagano, K., Enomoto, M., Yamanouchi, K., Aiso, S. and Katagiri, T. 1988. Toxicologic pathology of upper respiratory tract. J. Toxicol. Pathol. 1: 115-127.

10. National Toxicology Program (NTP). 1990. Toxicology and carcinogenesis studies of 2,6-xylidine (2,6-dimethylaniline) in Charles River CD rats (feed studies). NTP Technical Report No.278. U.S. Department of Health and Human Services, Public Health Service, National Institutes of Health, Bethesda.

11. Pino, M. V., Valerio, M. G., Miller, G. K., Larson, J. L., Rosolia, D. L., Jayyosi, Z., Crouch, C. N., Trojanowski, J. Q. and Geiger, L. E. 1999. Toxicologic and carcinogenic effects of the type IV phosphodiesterase inhibitor RP 73401 on the nasal olfactory tissue in rats. Toxicol. Pathol. 57: 383-394.

12. Rudd, C. J., Mitchell, A. D. and Spalding, J. 1983. L5178Y mouse lymphoma cell mutagenesis assay of coded chemicals incorporating analyses of the colony size distributions. Environ. Mutagen. 5: 419.

13. Uraih, L. C. and Maronpot, R. R. 1990. Normal histology of the nasal cavity and application of special techniques. Environ. Health Perspect. 85: 187-208.

14. Uraih, L. C., Talley, F. A., Mitsumori, K., Gupta, B. N., Bucher, J. R. and Boorman, G. A. 1987. Ultrastructural changes in the nasal mucosa of Fischer 344 rats and $\mathrm{B} 6 \mathrm{C} 3 \mathrm{~F} 1$ mice following an acute exposure to methyl isocyanate. Environ. Health Perspect. 72: 77-88.

15. WHO 1996. Toxicological evaluation of certain veterinary drug residues in food. WHO Food Additives Series: 38. World Health Organization, Geneva.

16. WHO 1998. Evaluation of certain veterinary drug residues in food. WHO Technical Report Series: 876. World Health Organization, Geneva.

17. Zimmer, D., Mazurek, J., Petzold, G. and Bhuyan, B. K. 1980. Bacterial mutagenicity and mammalian cell damage by several substituted anilines. Mutat. Res. 77: 317-326. 